This paper is a postprint of a paper submitted to and accepted for publication in IET Generation, Transmission and Distribution (GTD) and is subject to IET copyright. The copy of record is available at [http://digital-library.theiet.org/]" and the paper is available through IEEE Xplore at [http://ieeexplore.ieee.org/xpls/abs_all.jsp?arnumber=4907255]

\title{
Frequency and fundamental signal measurement algorithms for distributed control and protection applications
}

\section{A.J. Roscoe, G.M. Burt and J.R. McDonald}

\begin{abstract}
:
Increasing penetration of distributed generation within electricity networks leads to the requirement for cheap, integrated, protection and control systems. To minimise cost, algorithms for the measurement of $\mathrm{AC}$ voltage and current waveforms can be implemented on a single microcontroller which also carries out all other protection and control tasks, including communication and data logging. This limits the frame rate of the major algorithms, although Analogue to Digital Converters (ADCs) can be over-sampled using peripheral control processors on suitable microcontrollers. Measurement algorithms also have to be tolerant of poor power quality which may arise within grid-connected or islanded (e.g. emergency, battlefield or marine) power system scenarios. This paper presents a "Clarke-FLL hybrid" architecture which combines a 3-phase Clarke transformation measurement with a Frequency Locked Loop (FLL). This hybrid contains suitable algorithms for the measurement of frequency, amplitude and phase within dynamic 3-phase AC power systems. The Clarke-FLL hybrid is shown to be robust and accurate, with harmonic content up to and above $28 \% \mathrm{THD}$, and with the major algorithms executing at only 500 samples per second. This is achieved by careful optimisation and cascaded use of exact-time averaging techniques, which prove to be useful at all stages of the measurements: from DC bias removal through low-sample-rate Fourier analysis to sub-harmonic ripple removal. Platform-independent algorithms for three-phase nodal power flow analysis are benchmarked on three processors including the Infineon TC1796 microcontroller, on which only $10 \%$ of the $2000 \mu$ s frame time is required, leaving the remainder free for other algorithms.
\end{abstract}


This paper is a postprint of a paper submitted to and accepted for publication in IET Generation, Transmission and Distribution (GTD) and is subject to IET copyright. The copy of record is available at [http://digital-library.theiet.org/]" and the paper is available through IEEE Xplore at [http://ieeexplore.ieee.org/xpls/abs_all.jsp?arnumber=4907255]

\section{$1 \quad$ Introduction}

This paper presents an architecture for the measurement of frequency, amplitude and phase within 3-phase AC power systems. The architecture is designed to fulfil a number of emerging requirements pertinent to distributed generation and microgrid control applications:

- The measurements must settle quickly; within $\sim 5$ cycles for a frequency or voltage measurement used by a droop controller, and within $\sim 2$ cycles for an amplitude measurement used for protective relaying. The measurements must be able to track rapid frequency changes up to $10 \mathrm{~Hz} / \mathrm{s}$ during islanded operation.

- The measurement of frequency should be able to ride through single-phase, two-phase and partial-depth three-phase faults indefinitely. The frequency measurement should also be able to ride through a full-depth three-phase fault for a configurable amount of time.

- To enable deployment on cheap microcontrollers, the major signal processing algorithms should be able to operate at a fixed frame rate, possibly clocking as slowly as 10 samples per cycle $(500 \mathrm{~Hz}$ for a $50 \mathrm{~Hz}$ power system; a frame time of $2000 \mu \mathrm{s})$. The execution time of the measurements should take much less than $2000 \mu \mathrm{s}$; this allows other protection and control applications, including communications and data logging applications, to share the same processor.

- The architecture and algorithm should operate with acceptable accuracy under the influence of significant levels of harmonics, inter-harmonics, unbalance, flicker, and instrumentation/ADC noise. A suitable target frequency measurement accuracy (ripple) is $\pm 0.005 \mathrm{~Hz}$. Voltage amplitude measurement accuracy (ripple) should be better than \pm 0.01 pu for 2 -cycle measurements (protective relaying) and better than $\pm 0.001 \mathrm{pu}$ for 5 -cycle measurements used as inputs to reactive-power droop controllers.

The simplest examples of algorithms for single-phase amplitude measurement are the 2-sample and 3-sample techniques for amplitude measurement [1], which give poor results 
This paper is a postprint of a paper submitted to and accepted for publication in IET Generation, Transmission and Distribution (GTD) and is subject to IET copyright. The copy of record is available at [http://digital-library.theiet.org/]" and the paper is available through IEEE Xplore at [http://ieeexplore.ieee.org/xpls/abs_all.jsp?arnumber=4907255]

in the presence of harmonic or noise contamination. Amplitudes can also be measured using very slow (under-sampled) rates as presented by [2], although these techniques rely on more static network conditions than the requirements of this paper allow. Amplitude measurements can also be made using Fourier-based algorithms, with sensible implementations available in [3]. These algorithms function at low sample rates down to 10 samples-per-cycle, and this paper presents methods for substantially decreasing the output ripple due to harmonic contamination and interpolation at such low sample rates.

The simplest frequency measurement is the zero-crossing method, which is adapted in [4] to reduce the errors due to interpolation at low sample rates. The final result, however, is still subject to relatively large errors up to $0.05 \mathrm{~Hz}$ under test conditions of $0.3 \mathrm{pu} 3^{\text {rd }}$ harmonic and a $30 \mathrm{~dB}$ Signal to Noise ratio (SNR), using a $1440 \mathrm{~Hz}$ sample rate. Other methods for "instantaneous" frequency measurement of single-phase waveforms such as [5] are also very sensitive to harmonics and noise, and require pre-filters of significant latency in order to reject these effects. In [6] an algorithm is designed to be immune to harmonic contamination, but still provides relatively poor performance $(0.1 \mathrm{~Hz}$ accuracy) even at very high sample rates of nearly $30 \mathrm{kHz}$.

A relatively simple and effective method of measuring frequency in a three-phase power system is to use the Clarke transformation to obtain the $\mathrm{ABO}$ vector, and then to analyse the rotation of the $A B$ vector.

$$
\left[\begin{array}{l}
A \\
B \\
0
\end{array}\right]=\left[\begin{array}{ccc}
\frac{2}{3} & \frac{-1}{3} & \frac{-1}{3} \\
0 & \frac{1}{\sqrt{3}} & \frac{-1}{\sqrt{3}} \\
\frac{1}{3} & \frac{1}{3} & \frac{1}{3}
\end{array}\right] *\left[\begin{array}{l}
V_{a} \\
V_{b} \\
V_{c}
\end{array}\right]
$$

For a balanced three-phase voltage set, the vector $\mathbf{A B}$ rotates steadily at the system frequency, but in the presence of harmonics, inter-harmonics, flicker, unbalance and noise the rotation is not constant. Such a frequency measurement method is proposed in [7], because it can seamlessly ride through any single-phase fault, during which time the $A B$ 
This paper is a postprint of a paper submitted to and accepted for publication in IET Generation, Transmission and Distribution (GTD) and is subject to IET copyright. The copy of record is available at [http://digital-library.theiet.org/]" and the paper is available through IEEE Xplore at [http://ieeexplore.ieee.org/xpls/abs_all.jsp?arnumber=4907255]

vector trajectory changes from a circle to an ellipse [8]. However, AB vector angle change in [7] is only measured on a sample-by-sample basis and not averaged over a half-cycle or full-cycle period, and thus must be smoothed substantially to remove the ripple effects introduced by harmonics and unbalance. Additionally, the AB vector angle change in [7] is estimated using a Cartesian coordinate system instead of an atan2() function; this introduces problems during large changes of signal amplitude. This mathematical simplification avoids the computation time of the atan2() function, in a similar manner to the avoidance of the $\sin ()$ and $\cos ()$ functions in [9], which was a sensible approach in the 1990s. Modern microcontrollers such as the Infineon TC1796 can compute atan2() in less than $0.75 \mu \mathrm{s}$ and sine/cosine in $0.6 \mu \mathrm{s}$, times which are not much greater than the execution time of lookup tables $(0.5 \mu \mathrm{s})$ or other approximated algorithms. Thus, this paper proposes that trigonometric functions should be used where necessary inside algorithms, and not strictly avoided.

Attempts to measure single-phase signals can also be made using a rotating vector approach using signal-splitting and $1 / 4$-cycle delaying algorithms such as presented in [10], although this method produces large ripples due to discretised errors in the signal delay implementation and in the presence of harmonics. Wavelet transforms have been used in [11] to analyse single-phase signals, although [11] presents very poorly coded Fourier transform results for comparison, and does not assess errors due to noise. The sample rate at $600 \mathrm{~Hz}$ approaches the $500 \mathrm{~Hz}$ requirement, although only harmonics up to the $6^{\text {th }}$ are applied in the test scenarios. The measured frequency error during a voltage dip to $0.4 \mathrm{pu}$ is also shown to be as high as $0.5 \%(0.25 \mathrm{~Hz})$, which is unacceptable.

Many Phase Locked Loops (PLLs) of the single-phase and three-phase variety have been presented, most with the aim of controlling solid-state inverters. A useful concept is that of normalising the response such that the dynamics of the frequency measurement are unaffected by the amplitude of the input signal $[12,13]$. A useful foundation for the work presented in this paper is [14] which recognises the enormous value of averaging over exactly one single signal period using [15]. Also in [14], a $2^{\text {nd }}$ harmonic cancellation 
This paper is a postprint of a paper submitted to and accepted for publication in IET Generation, Transmission and Distribution (GTD) and is subject to IET copyright. The copy of record is available at [http://digital-library.theiet.org/]" and the paper is available through IEEE Xplore at [http://ieeexplore.ieee.org/xpls/abs_all.jsp?arnumber=4907255]

scheme is devised which reduces the interpolation errors (of the fundamental) due to low sample rate measurement. In this paper, an alternative $1 \frac{1}{2} 2$-cycle measurement scheme is proposed which provides better dynamic performance and better rejection of higher order harmonics.

The paper presents an overview of a new "Clarke-FLL hybrid" measurement architecture and algorithms to meet the strict requirements listed above. More in-depth details of the actual code, including Simulink screenshots, are available in [16]. The target of this work is not to design a PLL, but to measure frequency and amplitude/phase. However, many of the concepts presented in this paper can also be applied to PLL design.

\section{Amplitude, measurements \\ phase and \\ frequency}

\subsection{Averaging signals over exact-time periods}

The benefit of being able to average (or integrate) a signal over an exact timeframe (generally not equal to an exact number of sample intervals) cannot be overstated. Normally, the most useful timeframe is one cycle period as used in [3] and [14]. However, the work presented below uses several other averaging intervals, usually multiples of half-periods, but sometimes other (longer) time lengths to remove sub-harmonic ripple. The most obvious benefits are to obtain accurate, low-ripple outputs from single-cycle Fourier correlations or measurements of elliptical $A B$ vector rotation over half or one cycle. Such ripples in the results of the works [7, 9, 10,12,13] could be removed by the use of such exact-time averaging techniques. The difficulty with exact-time averaging within a fixed-frame-rate system is that the desired time period to average over is normally not an integral multiple of the frame time. Thus, interpolation is required. The algorithm of [15] addresses this issue but contains two drawbacks. Firstly, the algorithm output represents the result of an averaged time period which does not always end at the current sample instant. The result is lagged by a time length which is dynamically variable between 0 and the sample interval $\Delta t$. The second issue is simply that the sub-algorithm [17] is not fully optimised for speed or reliability within embedded code. 
This paper is a postprint of a paper submitted to and accepted for publication in IET Generation, Transmission and Distribution (GTD) and is subject to IET copyright. The copy of record is available at [http://digital-library.theiet.org/]" and the paper is available through IEEE Xplore at [http://ieeexplore.ieee.org/xpls/abs_all.jsp?arnumber=4907255]

Fig. 1 shows how the first point can be addressed. The new, improved algorithm continually adds trapezoidally integrated segments to an accumulator using the newest two samples. The approximate signal average is then the difference between the current value of this accumulator and its value $n$ samples ago. To account for time intervals not equal to multiples of the sample interval $\Delta t$, a trapezoidal area calculated from the oldest 2 samples using linear interpolation must be subtracted from the result. This algorithm requires the use of 2 rolling memory buffers: one for the accumulated area and one for the sampled values, whereas [15] only requires 1 buffer.

To optimise the speed and robustness of the algorithm compared to [15], three further enhancements are required: 1) Given a single estimate of signal frequency, which determines the required averaging time, a single "Part A" pre-computation block determines the number of delay samples required and the factors required for linear interpolation. This can be re-used by many "Part B" averaging stages which are required to average different signals over the same averaging time. 2) The delay buffers are optimised by careful coding within Simulink "S functions" (fully “in-lined") using pointer arithmetic. Execution time on the TC1796 microcontroller is reduced to $0.25 \mu$ s per buffer compared to $0.75 \mu$ s for [17]. 3) To avoid integrator wind-up and loss of precision, two independent trapezial integration stages are used in parallel, with a separate delay buffer required for each to form the definite integral. These are used in a tick-tock fashion which allows resetting of the integrators to zero at regular intervals. The total number of delay buffers required per averaging stage is thus increased further from 2 to 3 . This architecture is summarised in Fig. 2.

The architecture of Fig. 2. has been created in embedded code using both linear and $2^{\text {nd }}$-order interpolation for both the integration and interpolation stages. The $2^{\text {nd }}$-order code can give better results in scenarios of low noise and low harmonic content (Fig. 3), but the advantage decreases when noise or harmonic content is high. Therefore, the 
This paper is a postprint of a paper submitted to and accepted for publication in IET Generation, Transmission and Distribution (GTD) and is subject to IET copyright. The copy of record is available at [http://digital-library.theiet.org/]" and the paper is available through IEEE Xplore at [http://ieeexplore.ieee.org/xpls/abs_all.jsp?arnumber=4907255]

linearly interpolated algorithm, being slightly simpler, is generally more appropriate. This averaging filter is a purely FIR (Finite Impulse Response) device.

\subsection{Single and $1 \frac{1}{2}$ cycle Fourier-based measurements}

To make a single-cycle Fourier measurement of the fundamental at sample rates down to 10 samples per cycle, [3] can be adapted by using the exact-time average block from section 2.1. The complex value $F$ representing the fundamental can be calculated using the following equation, implemented in the digital domain using the averaging (integrating) algorithm described in detail above.

$F=\frac{2}{T}\left[\int_{t_{0}-T}^{t_{0}} y(t) \cdot \sin (\phi) \cdot d t+j \cdot \int_{t_{0}-T}^{t_{0}} y(t) \cdot \cos (\phi) \cdot d t\right]$

where $\phi=2 \pi \cdot f \cdot t, f$ is the estimate of frequency, $t_{0}$ is "now" and $T$ is the integration time $(1 / f$ for a single-cycle measurement $)$.

The signal magnitude is then given by $|F|$ and the signal phase (relative to the correlating waveform) is given by $\theta=\angle F$. Notably, the "absolute phase" is then given by $(\theta+\phi)$ and the fundamental may be estimated by $|F| \cdot \sin (\theta+\phi)$. A packet of data containing the phase $(\theta+\phi)$, the frequency $f$ and an accurate timestamp (e.g. from a Global Positioning system) can be passed to distant protection/control systems. Upon receipt, the phase data can be compared to other similar data accurately, accounting for variable latencies in the communications channels.

Another useful modification to [3] is to pre-compute the sine and cosine terms for the current estimate of system frequency. These values can be re-used for many signals, reducing execution time. The Fourier algorithm has a 1-cycle settling time, reasonable attenuation of noise, and reasonably low ripple at the output due to interpolation error (see Fig. 3). The ripple at the output is zero if the input signal period is equal at an exact 
This paper is a postprint of a paper submitted to and accepted for publication in IET Generation, Transmission and Distribution (GTD) and is subject to IET copyright. The copy of record is available at [http://digital-library.theiet.org/]" and the paper is available through IEEE Xplore at [http://ieeexplore.ieee.org/xpls/abs_all.jsp?arnumber=4907255]

number of sample intervals $\Delta t$, but rises to a maximum if the signal period is equal to an odd number of half-sample intervals i.e. $m \Delta t / 2$ where $m$ is odd. The ripple also increases as the level of harmonic contamination rises.

To address the interpolation errors shown in Fig. 3, [14] proposes the use of $2^{\text {nd }}$ harmonic cancellation within the measurement algorithm. This works well for clean sinusoids, but not for signals containing higher-order harmonics. It also introduces an extra undesirable feedback loop into the analysis which affects the dynamic settling performance. An alternative method proposed in this paper is to apply a short subsequent section of averaging, using the exact-time averaging blocks. The design of the subsequent averaging filters is determined by the quality of the ripple to be removed. It can be shown that under input conditions consisting of DC offset, fundamental frequency $f$, un-aliased higher order harmonics, and/or an error in the estimate of signal frequency, that the ripple frequencies at the output of a single-cycle Fourier correlation are predominantly at multiples of $2 f$. Thus the ripple can be almost completely removed by a further stage of averaging of exactly one half-period (or multiples thereof), as shown in Fig. 4. This can be called a " $1+1 / 2$ " measurement system, and consists purely of Finite Impulse Response (FIR) filters. Similarly, the initial Fourier correlation can be calculated on a half-cycle basis, leading to ripples which predominantly fall at frequencies which are multiples of $f$. In this case, the ripples at the $1 / 2$-cycle output due to DC offset and even harmonics are very large. However, the ripples can be almost totally removed by subsequent averaging over exactly one cycle, leading to a " $1 / 2+1$ " system with virtually identical performance to the " $1+1 / 2$ " measurement system. This can be useful if the half-cycle output is desirable for very fast, (but approximate) measurements for relaying purposes.

Table 1 shows the worst case RMS ripple errors resulting due to $10 \% 3^{\text {rd }}$ harmonic (Twice that allowed under BS EN 50160[18]) with a sample rate of 10 samples per cycle. This shows that the performance of the purely FIR “ $1+1 / 2$ " system is, for practical purposes, as good or better than any equivalent system using $2^{\text {nd }}$ harmonic cancellation, although it is significantly simpler to implement. It also settles fully within $1 \frac{1}{2}$ cycles, whereas the $2^{\text {nd }}$ 
This paper is a postprint of a paper submitted to and accepted for publication in IET Generation, Transmission and Distribution (GTD) and is subject to IET copyright. The copy of record is available at [http://digital-library.theiet.org/]" and the paper is available through IEEE Xplore at [http://ieeexplore.ieee.org/xpls/abs_all.jsp?arnumber=4907255]

harmonic cancellation algorithm exhibits ringing following sudden signal changes.

\subsection{Attenuation of potentially aliased harmonics}

At such low sample rates as 10 samples per cycle within the core measurement algorithm, many harmonics of the input signal will be aliased. To counter this, three techniques can be used together. Firstly, a pair of simple cascaded low-pass filters (125Hz cutoff) can be implemented in hardware using economical components. The cutoff frequency should not be lowered too far to avoid excessive group delay. Secondly, many modern target platforms such as the Infineon TC1796 contain parallel peripheral processors. These can be used to over-sample ADCs and perform basic filtering. With the main algorithms executing at only $500 \mathrm{~Hz}$, the $5^{\text {th }}$ or higher harmonics may be aliased. The $9^{\text {th }}$ and $11^{\text {th }}$ cause most practical concern, being at relatively high levels within power systems, attenuated by as little as $22.9 \mathrm{~dB}$ in the two low-pass filters, and because they can alias back onto or near the fundamental with a $500 \mathrm{~Hz}$ sample rate. This can cause sub-harmonic measurement ripples/errors of up to $0.002 \mathrm{pu}$ RMS for a $9^{\text {th }}$ harmonic at $3 \%$, or $0.005 p u$ RMS for an $11^{\text {th }}$ harmonic at 7\%, these being twice the levels allowed by BS EN 50160 [18]. To attenuate the $9^{\text {th }}$ and $11^{\text {th }}$ harmonics, over-sampling and simple FIR filters can be used at either $3 \mathrm{kHz}$ or $1.5 \mathrm{kHz}$. The $3 \mathrm{kHz}$ version attenuates the $9^{\text {th }}$ and $11^{\text {th }}$ by $>20 \mathrm{~dB}$ and $>15 \mathrm{~dB}$ respectively, while the $1.5 \mathrm{kHz}$ version provides marginally $20 \mathrm{~dB}$ and $15 \mathrm{~dB}$ attenuation. The filter weights for the $3 \mathrm{kHz}$ version shown in Fig. 5 are $[0.238507,0.0614238,0.200069$, $0.200069,0.0614238,0.238507]$. The $1.5 \mathrm{kHz}$ version uses weights of $[0.348834,0.302331$, 0.348834]. An alternative approach is to place the filter notches at exactly $500 \mathrm{~Hz}, 1000 \mathrm{~Hz}$ etc. using equal weights (3-sample or 6 sample averaging). This attenuates the $9^{\text {th }}$ and $11^{\text {th }}$ almost as well, with the added benefit of notching out switching harmonics for active devices which inject at integer multiples of $500 \mathrm{~Hz}$. The amplitude and phase effect of the FIR filter at the actual signal frequency must be added to the known effect of the two $125 \mathrm{~Hz}$ low-pass filters when applying calibration corrections as shown in Fig. 7. The design of the FIR filter would need to be modified for higher frame rates than $500 \mathrm{~Hz}$ or different oversampling ratios. 
This paper is a postprint of a paper submitted to and accepted for publication in IET Generation, Transmission and Distribution (GTD) and is subject to IET copyright. The copy of record is available at [http://digital-library.theiet.org/]" and the paper is available through IEEE Xplore at [http://ieeexplore.ieee.org/xpls/abs_all.jsp?arnumber=4907255]

The third technique for minimising the effect of aliased harmonics is an adaptive ripple rejection post-filtering stage. This novel filter (see Fig. 6) has been designed specifically to reduce any remaining ripple from the measured signal(s), after all other processing. Due to the previous measurement stages, which include averaging over at least $1 \frac{112}{2}$ cycles, any remaining ripple is mostly sub-harmonic. Reducing such ripple at measurement outputs is desirable because this ripple can induce oscillations within control systems which rely on the measurements. The filter first removes both DC bias and linear slopes from the signal using the exact-time averaging algorithm, and then searches for the fundamental frequency of the remaining ripple using a variable-frequency low-pass filter followed by a zero-crossing detector. The estimated ripple frequency is filtered using a slew-rate filter and this determines the averaging time required to best remove the ripple (and its harmonics). Additional code detects rapid changes in the signal. When this occurs, the filter switches into a direct pass-through mode with zero latency (at the expense of higher ripple/noise). The threshold for transient detection on voltage amplitude can be set as low as $\pm 0.005 \mathrm{pu}$ for the measurement system and (steady state) test conditions described in this paper, without causing spurious transient detections. This means that an adaptive ripple filter with substantial averaging time (up to 3.5 cycles, $70 \mathrm{~ms}$ ), can be used at all times, because its latency will drop to zero during any significant transient events. Such an averaging time can completely remove all sub-harmonics down to $14.3 \mathrm{~Hz}$. If higher-order harmonics alias very closely to the actual signal frequency, within $14 \mathrm{~Hz}$, then the ripple removal filter will not be able to completely remove the sub-harmonic ripple. The extreme of this is a harmonic which aliases exactly onto the fundamental, which will produce a relatively benign DC offset to the result. The worst case would be measurement output ripples in the region of 0.1 to $1 \mathrm{~Hz}$ which might contribute to sub-harmonic power system oscillations if fed back through droop controllers. For this reason, the $125 \mathrm{~Hz}$ low-pass filters and 3x/6x over-sampled ADC are the front line of defence against aliased harmonics.

\subsection{General measurement architecture}

The combination of all the techniques described in sections 2.1-2.3 leads to the general measurement architecture shown in Fig. 7. The DC bias removal consists of 2 cascaded 
This paper is a postprint of a paper submitted to and accepted for publication in IET Generation, Transmission and Distribution (GTD) and is subject to IET copyright. The copy of record is available at [http://digital-library.theiet.org/]" and the paper is available through IEEE Xplore at [http://ieeexplore.ieee.org/xpls/abs_all.jsp?arnumber=4907255]

averaging filters of length one cycle each, to calculate the DC bias which is subtracted from the signal. The latency of this filter is thus zero, and its performance in this application surpasses that of a high-pass filter. The calculation and implementation of the calibration corrections, and the sequence/power-flow calculation algorithms cannot be presented here for reasons of brevity. It should be noted, however, that the sequence and power-flow analysis can be completed exactly without any further evaluations of sine or cosine by careful treatment of the averaged data from the Fourier transforms.

The cascaded FIR averaging stages both within and subsequent to the Fourier correlations provide excellent attenuation of noise and high-frequency inter-harmonics. A cascaded pair or triplet of FIR averaging filters provides better attenuation of Gaussian noise than a single FIR filter of equivalent length or an Infinite impulse response (IIR) low-pass filter of equivalent latency, due to the positioning of the transfer function zeros and the convolution of the rectangular impulse functions.

To measure amplitude/phase of voltage or current sets, given an estimate frequency, the architecture of Fig. 7 can be used directly, with a total measurement latency of $\sim 5.5$ cycles during steady state conditions and $\sim 2$ cycles $(40 \mathrm{~ms})$ during transients. These times are made up of $<1 / 2$ cycle due to the $125 \mathrm{~Hz}$ low-pass filters, $1 / 24^{\text {th }}$ cycle due to the $3 \mathrm{kHz}$ 6-tap FIR filter, 11/2 cycles for the Fourier and half-cycle averaging stages, and up to $3 \frac{1}{2}$ cycles (70ms average time) within the adaptive ripple removal.

\subsection{Clarke-FLL hybrid measurement}

To measure frequency together with amplitude and phase, the general architecture of Fig. 7 can be used as the basis for a complex "Clarke-FLL hybrid" measurement, shown in Fig. 8. This is a simplified diagram and the entire algorithm is a significant piece of Simulink coding, fully presented in [16]. A frequency measurement based upon a Clarke transformation is first used. This provides very fast settling over a very wide frequency range covering DC to the Nyquist frequency. Following application of measurable system voltages after an outage, the 2-cycle averaging filters individually switch in at set times of 
This paper is a postprint of a paper submitted to and accepted for publication in IET Generation, Transmission and Distribution (GTD) and is subject to IET copyright. The copy of record is available at [http://digital-library.theiet.org/]" and the paper is available through IEEE Xplore at [http://ieeexplore.ieee.org/xpls/abs_all.jsp?arnumber=4907255]

$100 \mathrm{~ms}$ and $160 \mathrm{~ms}$. This provides a 1 -cycle response initially, which then switches to a 3 cycle and 5-cycle response as appropriate. Given a three-phase voltage signal with low unbalance, this measurement has an almost entirely FIR type response, due to the steady rotation of the $A B$ vector. The measurement can fail during 2-phase faults due to collapse of the $A B$ vector trajectory [8], and cannot provide amplitude/phase measurements of the 3 phases. Measurement validity can be ascertained by placing suitable limits on the sample-to-sample angular rotation velocity of the $A B$ vector.

The Clarke's frequency measurement is used as a seed for a Frequency Locked Loop (FLL). The FLL contains 11/2-cycle Fourier amplitude/phase measurements of the three phase voltages. Frequency is detected for each of the 3 phases by the rate of phase change and then averaged further over $3 \frac{1}{2}$ cycles. A weighted average from the 3 phases then reveals a measurement of system frequency, which is fully tolerant to two-phase faults/transients and large levels of unbalance. The adaptive sub-harmonic ripple filtering is then applied, which switches out during fast-moving frequency transients. During the deepest three-phase voltage transients, both the Clarke transformation and FLL may be unable to obtain a valid measurement of frequency. This is due to low SNR and post-fault ringing within the power system itself. In this case, a temporary ride-through action can be initiated which holds frequency outputs to the last good values (taken from before the transient) and also pre-loads the $3 \frac{1}{2}$ cycle frequency averaging filters with these values. Similar averaging pre-load action takes place during seeding of the FLL's frequency with the value from the Clarke's algorithm. Seeding occurs when the FLL is not well locked but the fast-settling Clarke transform based measurement is valid. During transient events, an FLL without seeding would behave with an IIR response due to the Fourier measurement and feedback loop characteristics. Although the response would be more desirable than that of PLL(s), it is preferable to avoid this effect by using seeding from the Clarke's measurement. This combination provides an extremely fast settling and robust measurement algorithm hybrid. 
This paper is a postprint of a paper submitted to and accepted for publication in IET Generation, Transmission and Distribution (GTD) and is subject to IET copyright. The copy of record is available at [http://digital-library.theiet.org/]" and the paper is available through IEEE Xplore at [http://ieeexplore.ieee.org/xpls/abs_all.jsp?arnumber=4907255]

\section{Results and benchmarking}

The Clarke-FLL hyrid algorithm has been coded in MATLAB Simulink which provides platform independence. The code can be used directly in simulation, converted into a large "S function", or built into embedded C code for virtually any target microcontroller or other platform. Subsidiary "S functions" are used where appropriate to improve the speed of certain key functions, most notably the delay buffer blocks. The entire code for the Clarke-FLL hybrid of Fig. 8 (including 6 adaptive ripple-removal filters on the three-phase voltage and current outputs) contains $1 \sin (), 1 \cos (), 4 \operatorname{atan} 2(), 6 \operatorname{sqrt}()$, and 123 delay buffers. The total algorithm execution time (for the 500Sa/s frame rate algorithm) is $141 \mu \mathrm{s}$ on the Infineon TC1796 microcontroller $[19,20]$ when the program is stored in the on-board flash memory. The delay buffers account for approximately $0.25 \mu \mathrm{s}$ each, thus in total $35 \mu \mathrm{s}$ or $25 \%$ of the execution time. At a frame interval of $2000 \mu$ s, the algorithm requires $7 \%$ of the frame time. Extending the operation to measure both voltage and current, with full sequence and power flow analysis, but limiting the requirement for ripple-removal filters to 6 , can be achieved in $193 \mu \mathrm{s}$, less than $10 \%$ of the frame time. This same algorithm has been implemented on the legacy MVME5100 [21] and the newer MVME5500 [22] processors with execution times of 631 and $71 \mu$ s respectively. Adding further ripple-removal filters can lead to non-linear execution time increments on the TC1796, because total data memory use then exceeds $48 \mathrm{kB}$ which requires paging between multiple RAM areas. The MVME processors have much larger memory spaces and do not show this symptom.

The performance of the Clarke-FLL hybrid for frequency measurement is shown in Fig. 9, compared to the MATLAB SimPowerSystems (SPS) 3-phase PLL and the weighted average (weights set by measured phase magnitudes) of a set of 3 PLLs of the Jovcic design [14]. These two competing solutions have been presented here for two reasons. Firstly, they can easily be recreated by other researchers as benchmarks, due to the common availability of SPS and the detail of [14]. Secondly, these two methods present better frequency measurement accuracy then the other references $[2,4-7,9,10,12,13]$, particularly in the presence of harmonic contamination. The SPS PLL contains a single-cycle average of $\mathrm{q}$ 
This paper is a postprint of a paper submitted to and accepted for publication in IET Generation, Transmission and Distribution (GTD) and is subject to IET copyright. The copy of record is available at [http://digital-library.theiet.org/]" and the paper is available through IEEE Xplore at [http://ieeexplore.ieee.org/xpls/abs_all.jsp?arnumber=4907255]

error magnitude which allows it to tolerate unbalance and harmonics with reasonable effectiveness. The SPS PLL applies a $12 \mathrm{~Hz} / \mathrm{s}$ slew-rate limiter combined with a $2^{\text {nd }}$-order $25 \mathrm{~Hz}$ low-pass filter to the frequency measurement. The Jovcic PLLs also contain single-cycle averaging, together with a $1^{\text {st }}$-order $1.3 \mathrm{~Hz}$ low-pass filter within the frequency measurement.

A 60-second test waveform was used, incorporating noise and ADC quantisation (60dB SNR with 11-bit effective $A D C$ resolution) voltage dips/faults, frequency ramps to $10 \mathrm{~Hz} / \mathrm{s}, \mathrm{a}$ phase step, unbalance at 10\%, harmonic contamination to $28 \%$ THD spread across harmonics 2-40 using [18] and [23] as guides, and inter-harmonics at $2025 \mathrm{~Hz}(6.5 \%) \&$ $525 \mathrm{~Hz}(23 \%)$ to simulate worst-case signalling or inverter interference. The same anti-alias filters, oversampled ADCs, and FIR pre-filters were applied to all 3 methods to enable a fair comparison.

The Clarke-FLL hybrid shows excellent results, better than the other candidate algorithms in all cases apart from the phase jump test (Fig. 9e), where the SPS and Jovcic PLLs apply significant slew-rate and low-pass filtering to their results. However, these filters negatively impact the dynamic performance of the SPS and Jovcic PLLs. During initial signal application (Fig. 9a \& 9b), the FLL hybrid settles within $40-100 \mathrm{~ms}$, due to the use of the fast-settling Clarke measurement algorithm, whereas the SPS and Jovcic PLLs take up to $800 \mathrm{~ms}$ to lock and settle. In the presence of unbalance, harmonic and inter-harmonic contamination (Fig. 9c \& 9d), the FLL hybrid shows at least an order of magnitude lower steady-state error than the Jovcic PLLs, and significantly better performance than the SPS PLL. This is due to the combined use of the extra $1 / 2$-cycle averaging and the adaptive ripple removal filter, which prove to be more effective techniques than slew-rate limiting and/or low-pass filtering. Fig. $9 \mathrm{~h}$ shows that the FLL hybrid is able to track signals accurately over a wide frequency range with a high rate of change of frequency (ROCOF) up to $10 \mathrm{~Hz} / \mathrm{s}$ by using the Clarke measurement as a seed, whereas the SPS and Jovcic PLLs have lost lock before $t=39$ seconds due to the high frequency input, and they struggle or fail to regain lock between $t=39$ and $t=60$ seconds as frequency ramps quickly from $100 \mathrm{~Hz}$ 
This paper is a postprint of a paper submitted to and accepted for publication in IET Generation, Transmission and Distribution (GTD) and is subject to IET copyright. The copy of record is available at [http://digital-library.theiet.org/]" and the paper is available through IEEE Xplore at [http://ieeexplore.ieee.org/xpls/abs_all.jsp?arnumber=4907255]

to $10 \mathrm{~Hz}$ and back again. Fig. 9f shows how the fault ride-through action of the FLL hybrid allows it to exhibit less ringing than the PLLs during deep three-phase faults, and Fig. 9g shows how the FLL hybrid is virtually immune to full-depth single and two-phase faults. On Fig. 9g, the relatively poor performance of the Jovcic PLLs during the 2-phase fault is due to amplitude measurement instability within the 3 Jovcic PLLs running at the $2000 \mu$ s frame time, which feeds through the 3-phase weighted average block which was added for this test. The Jovcic PLLs appear to be much more stable at frame times of $500 \mu$ s or below.

The frequency measurement accuracy of the Clarke-FLL hybrid exceeds that of [24] for a class $A$ instrument $( \pm 0.01 \mathrm{~Hz})$ and generally achieves $\pm 0.005 \mathrm{~Hz}$, but at a fixed frame rate of only $500 \mathrm{~Hz}$ and requiring only $5 \frac{1}{2}-91 \frac{1}{2}$ cycles $(110-190 \mathrm{~ms})$ for full settling while [24] allows 500 cycles (10 seconds).

The amplitude measurement performance of the Clarke-FLL hybrid is shown in Fig. 10. The performance again meets that of [24] for a class $A$ instrument $( \pm 0.001 \mathrm{pu})$, apart from brief periods during the initial sudden onset or removal of harmonic content, but at a fixed frame rate of only $500 \mathrm{~Hz}$ and requiring only $2-5 \frac{1}{2}$ cycles $(40-110 \mathrm{~ms})$ for full settling while [24] allows 10 cycles $(200 \mathrm{~ms})$. It should be noted that here the fundamental component is measured, while [24] specifies an RMS voltage measurement. RMS voltages (which include harmonic content) cannot be measured accurately at such low frame rates due to the attenuation of the harmonic content in the anti-aliasing filters.

\section{Conclusions}

An architecture for a Clarke-FLL hybrid has been presented which allows measurement of frequency, amplitude and phase to world-class accuracy within 3-phase AC power systems, while the sample rate of the major algorithms can be reduced to only $500 \mathrm{~Hz}$. The measurement latencies are appropriate for protective and control functions within a microgrid scenario, incorporating autonomous filtering algorithms which adapt the latency as appropriate during transient or steady-state operation. To enable the target accuracy/ripple specification to be met for waveforms with harmonic content up to and 
This paper is a postprint of a paper submitted to and accepted for publication in IET Generation, Transmission and Distribution (GTD) and is subject to IET copyright. The copy of record is available at [http://digital-library.theiet.org/]" and the paper is available through IEEE Xplore at [http://ieeexplore.ieee.org/xpls/abs_all.jsp?arnumber=4907255]

beyond $28 \%$ THD, a $1.5 \mathrm{kHz}$ or $3 \mathrm{kHz}, 3 \mathrm{x}$ or $6 \mathrm{x}$ oversampled $\mathrm{ADC}$ must currently be used, together with a simple 3-tap or 6-tap FIR filter. This architecture can be realised on a modern microcontroller such as the Infineon TC1796.

The measurement of a 3-phase voltage/current set, including sequence and power flow analysis, can be executed in $193 \mu$ s on the Infineon TC1796, leaving the remaining $1807 \mu$ s available for other generator/microgrid measurement and control functions. This enables cheap deployment on multi-functional single-processor controllers, which can be used to enhance the security of supply, safety and efficiency of power systems.

All of the concepts, methods and algorithms described in this paper are presented to a greater level of detail in [16]. The algorithms are coded in a combination of Simulink and C-code (Simulink "S-functions" where appropriate for execution speed) and are fully robust for long-term real-time deployment. The Simulink “Real-Time-Workshop" and “Embedded Coder" features have proved to be an effective way of writing error-free code which can be tested in simulation on a PC and then deployed (without code modification) to real-time targets. Applications which allow the major algorithms (or just the initial Fourier correlations) to be clocked at higher than 10 samples per cycle will allow reduced noise/ripple on the measurement results compared to that presented in this paper, and ultimately the removal of the requirement for oversampled ADCs and FIR pre-filters. Alternatively, initial analysis shows that replacing the $2^{\text {nd }}$-order low-pass anti-alias filters with 4-pole Butterworth filters would also remove the requirement for oversampling, with little increase in latency and similar performance, provided these filters could be built in a repeatable manner using economical components.

The algorithmic designs and results from this work now provide an excellent foundation upon which to build more advanced microgrid control and protection applications. 
This paper is a postprint of a paper submitted to and accepted for publication in IET Generation, Transmission and Distribution (GTD) and is subject to IET copyright. The copy of record is available at [http://digital-library.theiet.org/]" and the paper is available through IEEE Xplore at [http://ieeexplore.ieee.org/xpls/abs_all.jsp?arnumber=4907255]

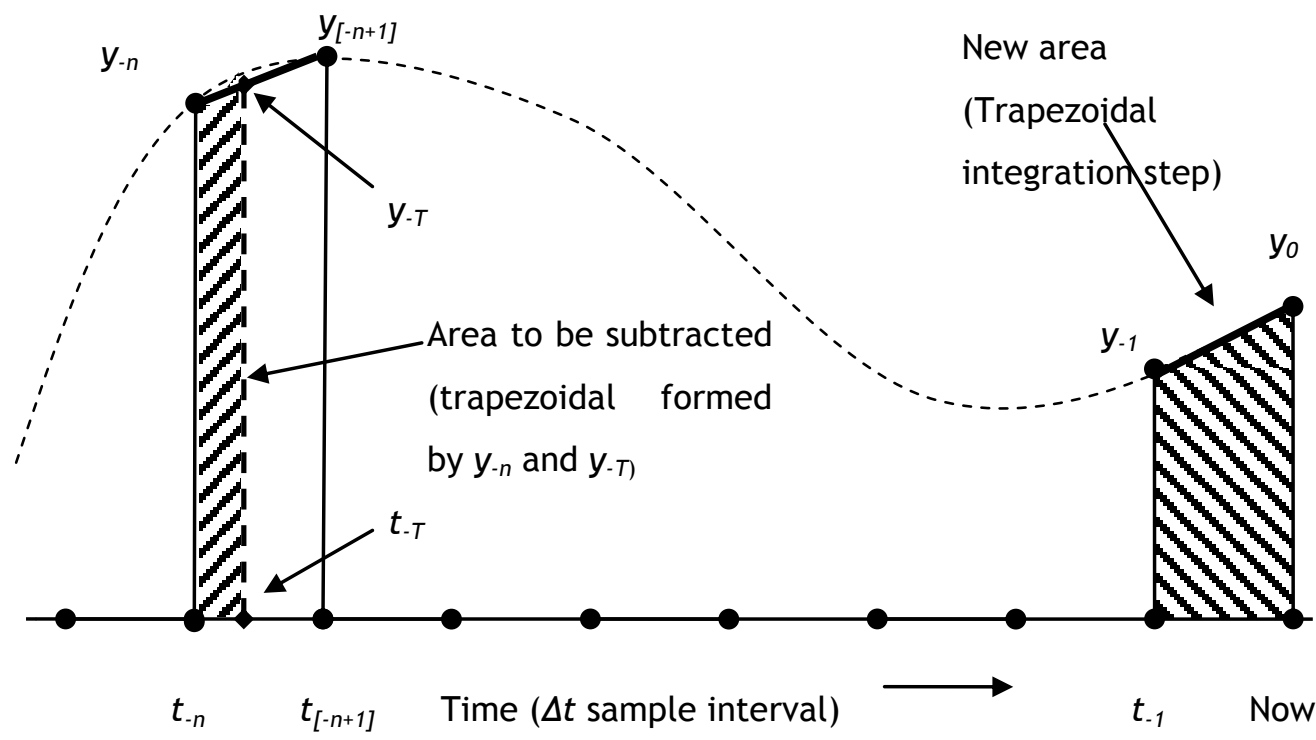

$\left(t_{0}\right)$

Fig. 1 Improved exact-time averaging technique

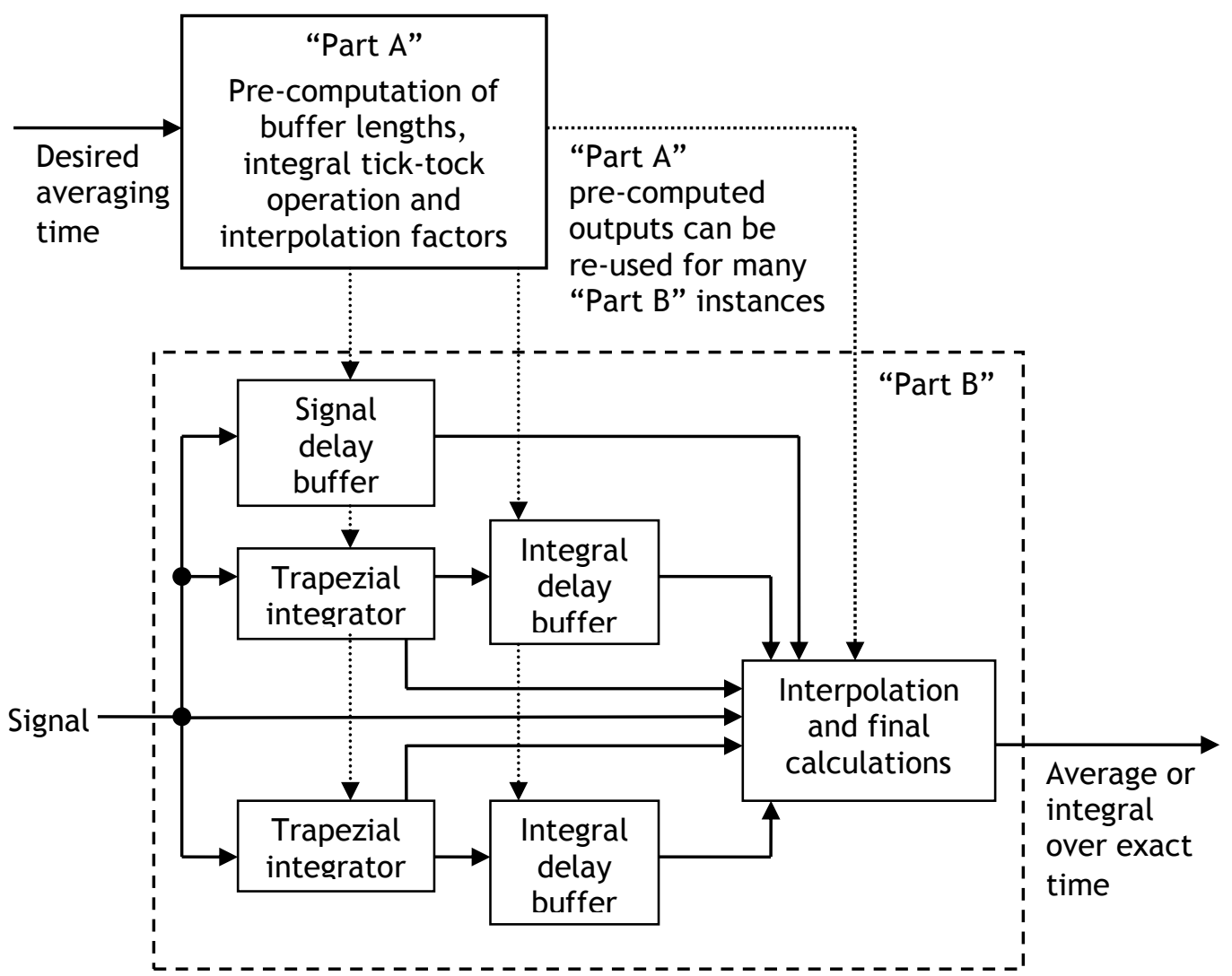

Fig. 2 Exact-time averaging architecture 
This paper is a postprint of a paper submitted to and accepted for publication in IET Generation, Transmission and Distribution (GTD) and is subject to IET copyright. The copy of record is available at [http://digital-library.theiet.org/]" and the paper is available through IEEE Xplore at [http://ieeexplore.ieee.org/xpls/abs_all.jsp?arnumber=4907255]

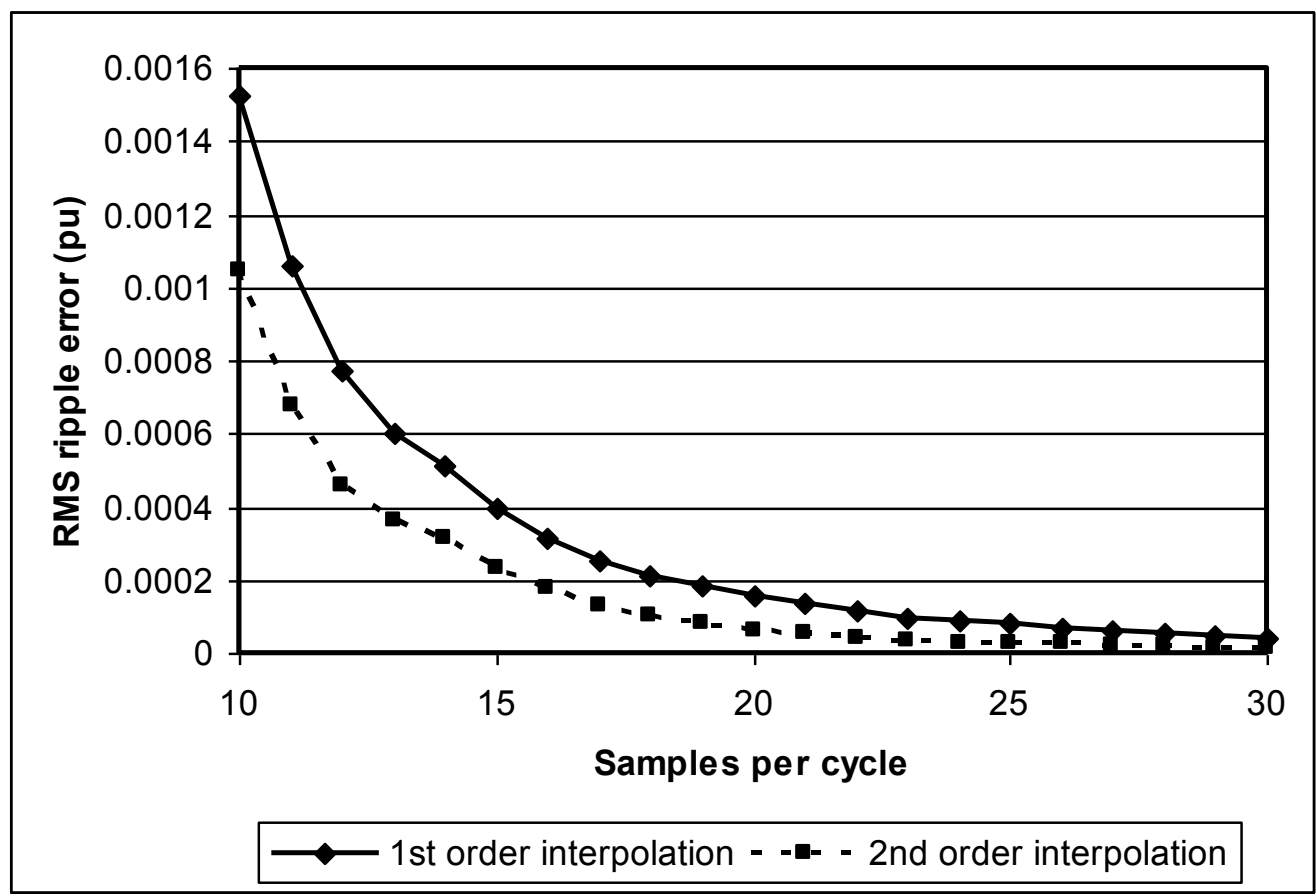

Fig. 3 Worst steady-state RMS ripple error for single-cycle Fourier amplitude measurement due to interpolation, for clean sinusoid input signals in the range 45$55 \mathrm{~Hz}$ with nominally 10 to 30 samples per cycle at $50 \mathrm{~Hz}$.

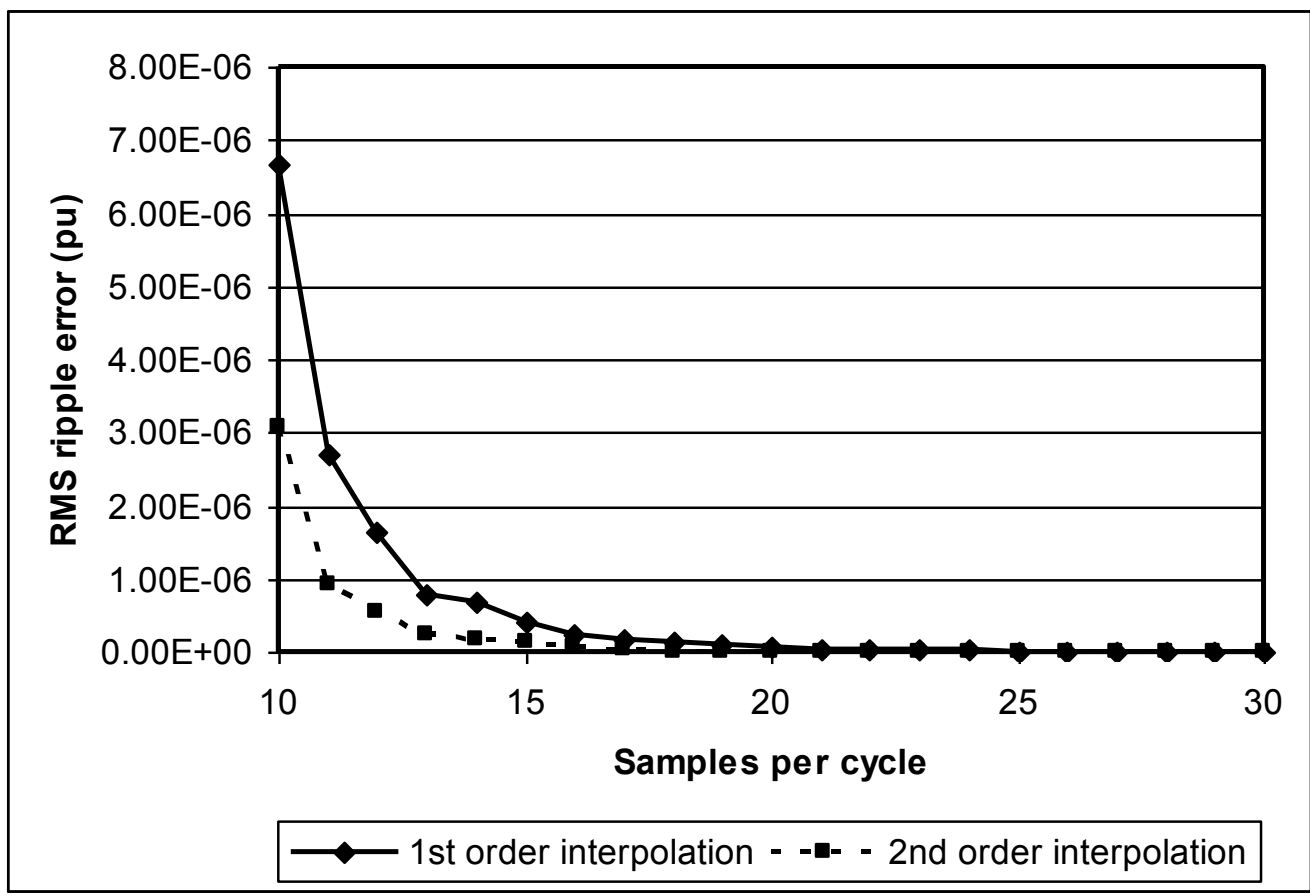

Fig. 4 Worst steady-state RMS ripple error for single-cycle Fourier amplitude measurement followed by $1 / 2$-cycle averaging, due to interpolation, for clean sinusoid input signals in the range $45-55 \mathrm{~Hz}$ with nominally 10 to 30 samples per cycle at $50 \mathrm{~Hz}$. 
This paper is a postprint of a paper submitted to and accepted for publication in IET Generation, Transmission and Distribution (GTD) and is subject to IET copyright. The copy of record is available at [http://digital-library.theiet.org/]" and the paper is available through IEEE Xplore at [http://ieeexplore.ieee.org/xpls/abs_all.jsp?arnumber=4907255]

\begin{tabular}{||c|c||}
\hline & $\begin{array}{c}\text { Worst steady-state RMS } \\
\text { ripple error (pu) }\end{array}$ \\
\hline 1 -cycle Fourier analysis & 0.0018 \\
\hline $\begin{array}{c}\text { "1+1/2" system. 1-cycle Fourier analysis followed by } \\
1 / 2 \text {-cycle averaging }\end{array}$ & 0.000036 \\
\hline 1 -cycle Fourier analysis with 2 ${ }^{\text {nd }}$ harmonic cancellation & 0.0012 \\
\hline $\begin{array}{c}1 \text {-cycle Fourier analysis with 2 } \\
\text { followed by } 1 / 2 \text {-cycle averaging }\end{array}$ & 0.000035 \\
\hline
\end{tabular}

Table 1: Worst steady-state RMS ripple errors due to $3^{\text {rd }}$ harmonic at $10 \%$, for signals in the range $45-55 \mathrm{~Hz}$ with nominally 10 samples per cycle at $50 \mathrm{~Hz}$.

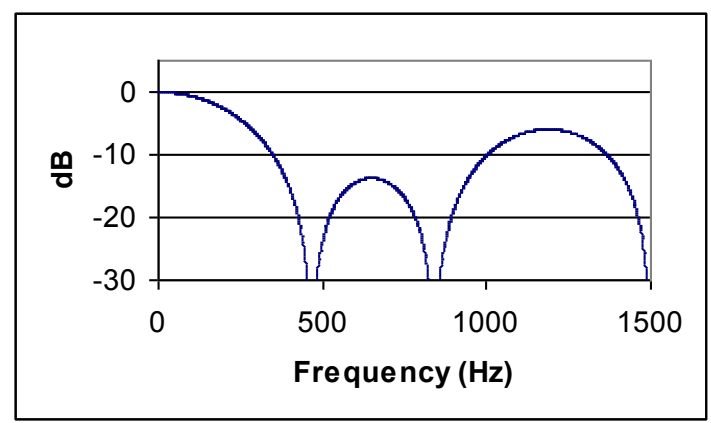

Fig. 5 Bode plot for $3 \mathrm{kHz}$ FIR filter: attenuation of $9^{\text {th }}$ and $11^{\text {th }}$ harmonic 
This paper is a postprint of a paper submitted to and accepted for publication in IET Generation, Transmission and Distribution (GTD) and is subject to IET copyright. The copy of record is available at [http://digital-library.theiet.org/]" and the paper is available through IEEE Xplore at [http://ieeexplore.ieee.org/xpls/abs_all.jsp?arnumber=4907255]

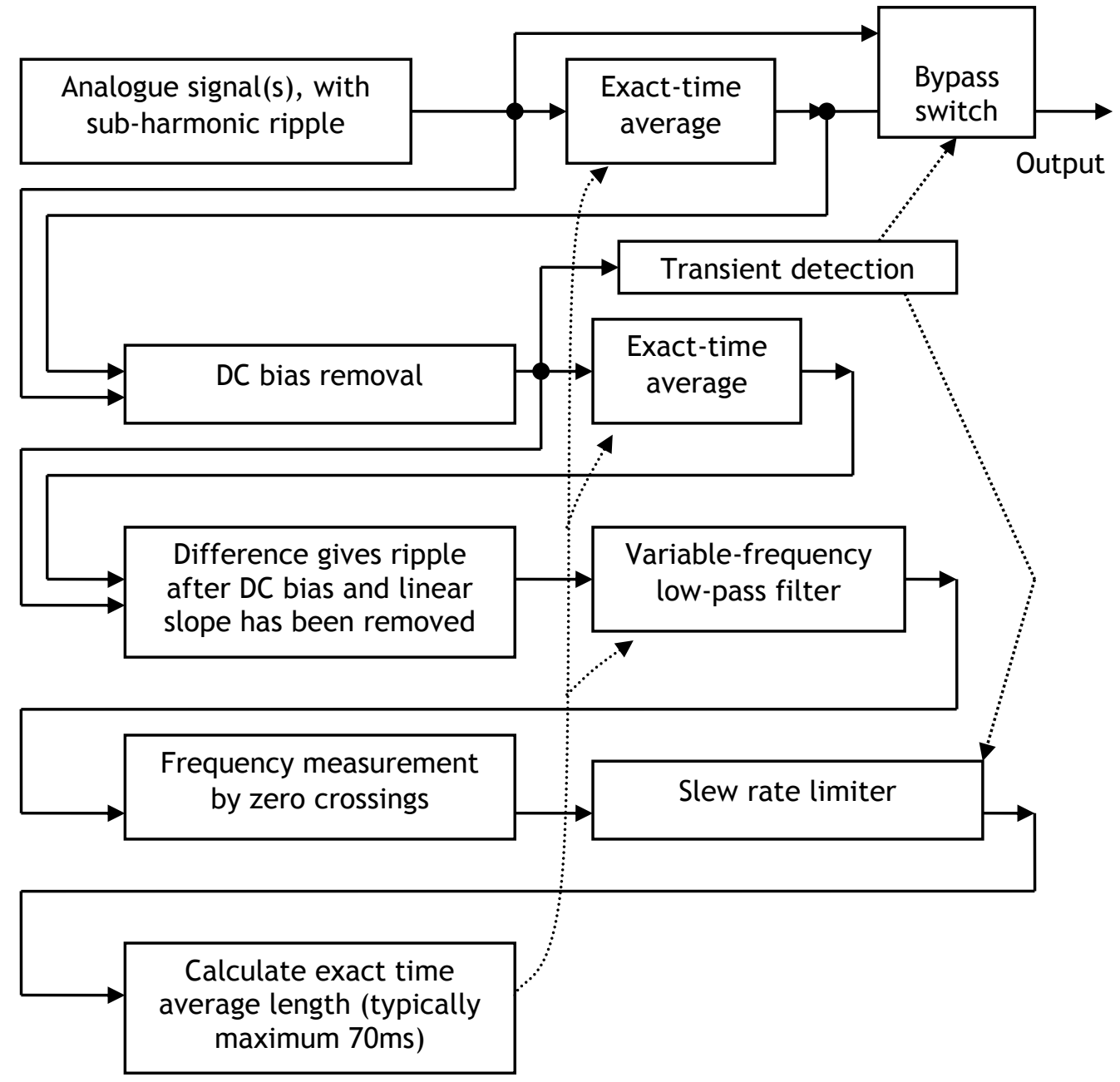

Fig. 6 Adaptive ripple removal filter architecture 
This paper is a postprint of a paper submitted to and accepted for publication in IET Generation, Transmission and Distribution (GTD) and is subject to IET copyright. The copy of record is available at [http://digital-library.theiet.org/]" and the paper is available through IEEE Xplore at [http://ieeexplore.ieee.org/xpls/abs_all.jsp?arnumber=4907255]

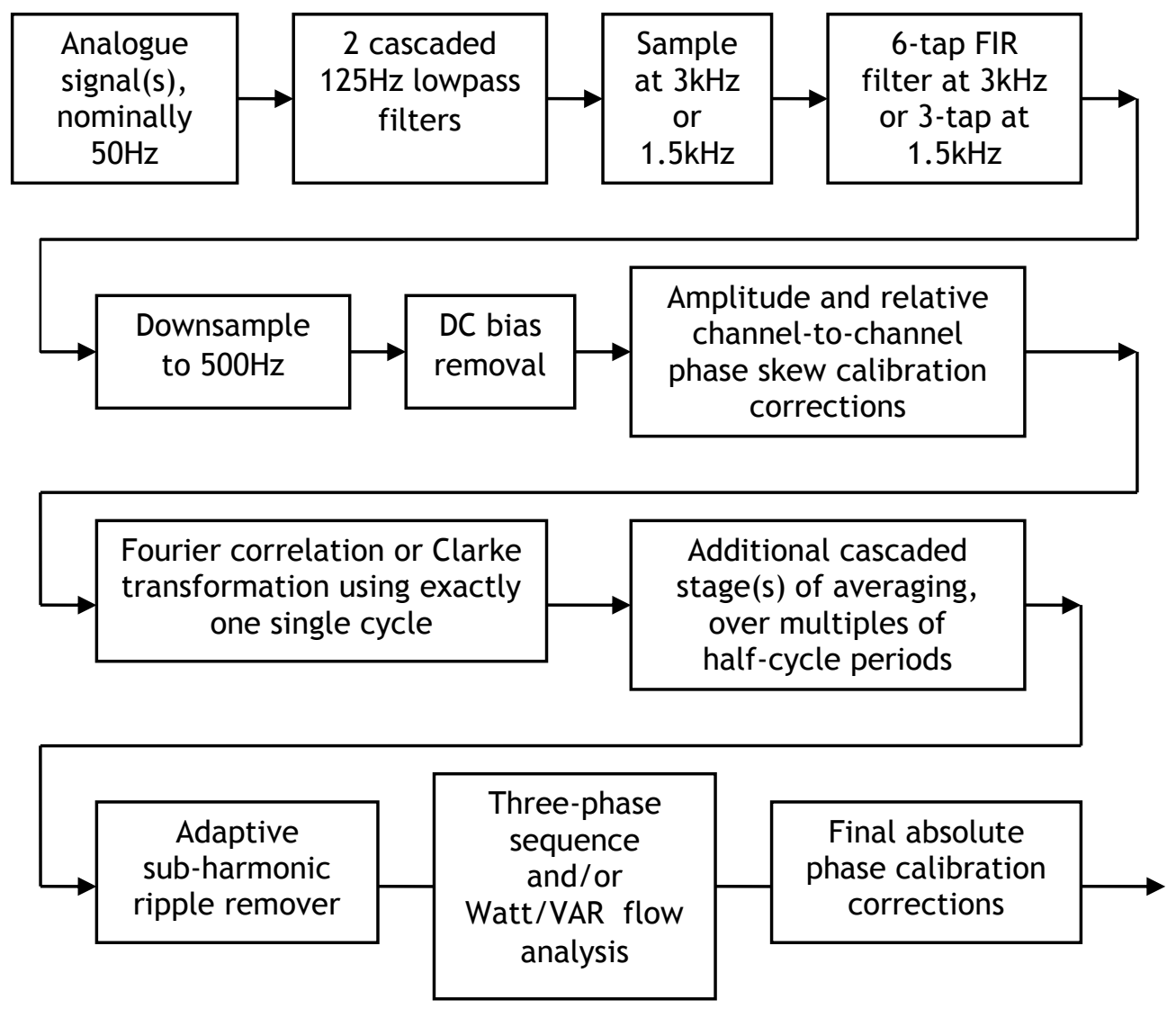

Fig. 7 General measurement architecture 
This paper is a postprint of a paper submitted to and accepted for publication in IET Generation, Transmission and Distribution (GTD) and is subject to IET copyright. The copy of record is available at [http://digital-library.theiet.org/]" and the paper is available through IEEE Xplore at [http://ieeexplore.ieee.org/xpls/abs_all.jsp?arnumber=4907255]

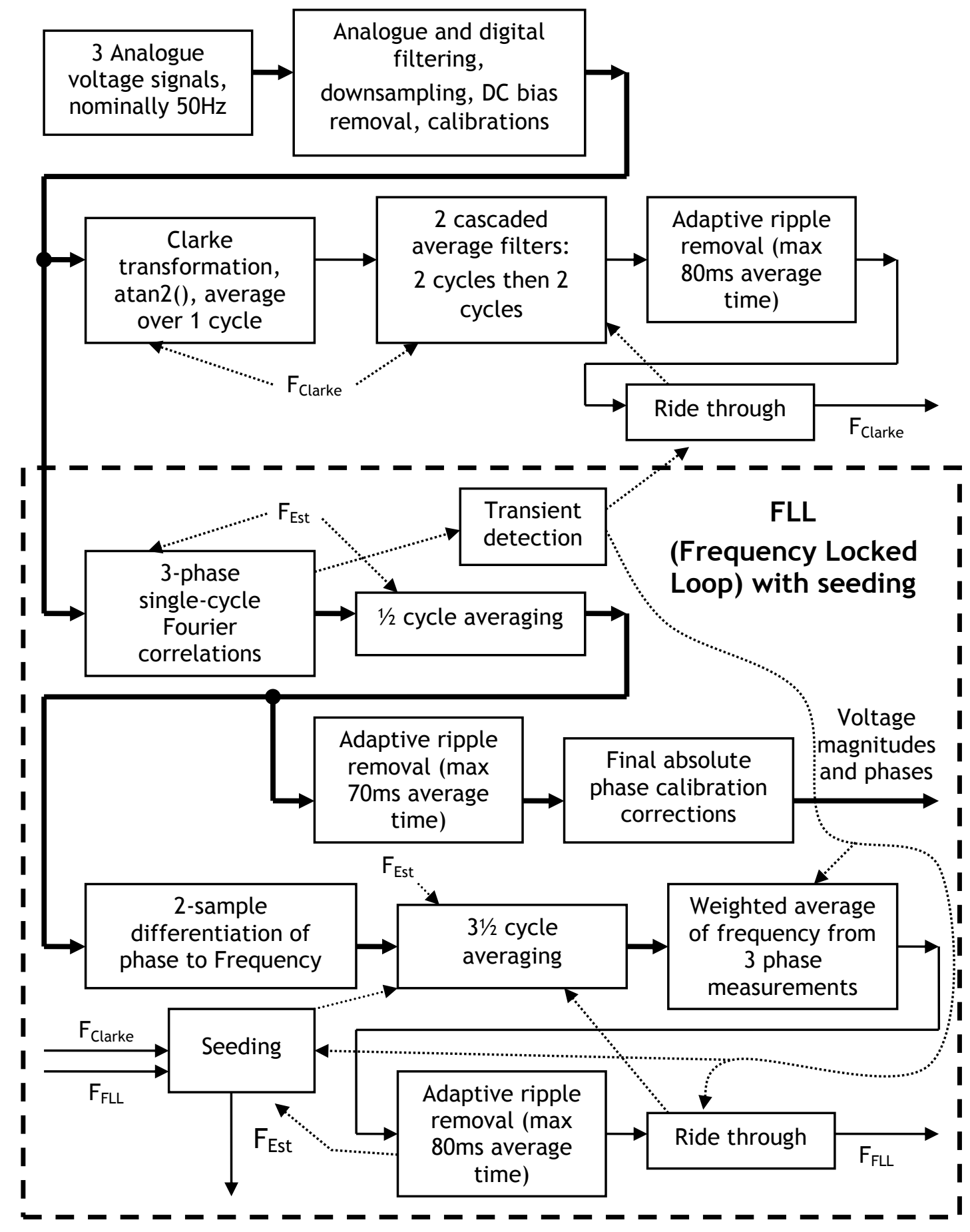

Fig. 8 Clarke-FLL hybrid architecture 
This paper is a postprint of a paper submitted to and accepted for publication in IET Generation, Transmission and Distribution (GTD) and is subject to IET copyright. The copy of record is available at [http://digital-library.theiet.org/]" and the paper is available through IEEE Xplore at [http://ieeexplore.ieee.org/xpls/abs_all.jsp?arnumber=4907255]
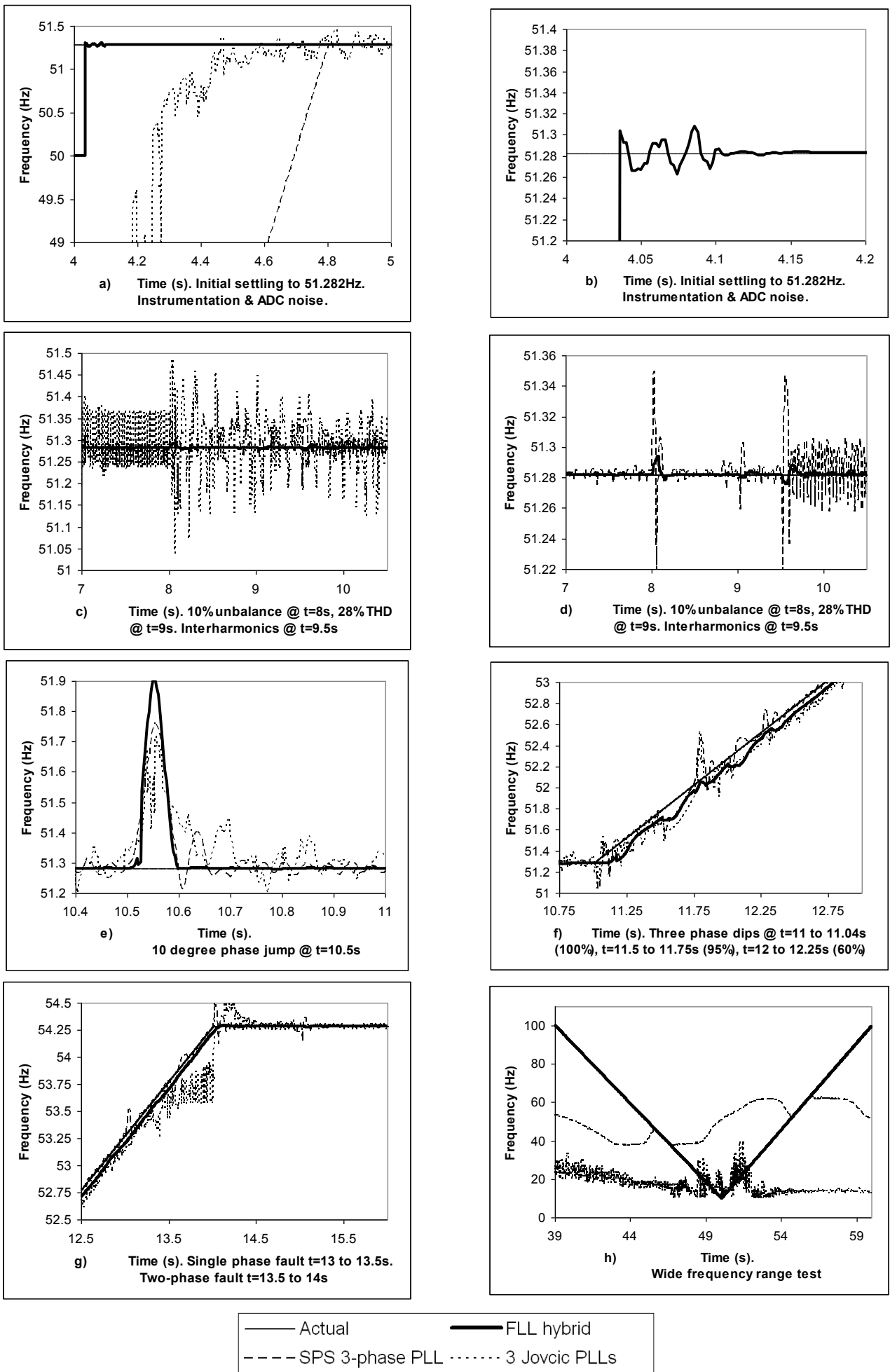

Fig. 9 Performance of FLL hybrid for frequency measurement

a) settling to $51.282 \mathrm{~Hz}$ at $\mathrm{t}=4 \mathrm{~s}$, with instrumentation and $\mathrm{ADC}$ noise

b) as a) but zoomed (SPS and Jovcic PLL results off the scale)

c) $51.282 \mathrm{~Hz}$. Unbalance added at $\mathrm{t}=8 \mathrm{~s}, 28 \% \mathrm{THD}$ at $\mathrm{t}=9 \mathrm{~s}$, \& inter-harmonics at $\mathrm{t}=9.5 \mathrm{~s}$

d) as c) but Jovcic PLL data not shown for clarity, and zoomed to show detail

e) $10^{\circ}$ phase jump at $\mathrm{t}=10.5 \mathrm{~s}$

f) $1 \mathrm{~Hz} / \mathrm{s}$ ramp with 3 -phase faults at $\mathrm{t}=11-11.04 \mathrm{~s}(100 \% \mathrm{dip}), 11.5-11.75 \mathrm{~s}$ (95\% dip), $12-12.25 \mathrm{~s}$ (60\% dip)

g) $1 \mathrm{~Hz} / \mathrm{s}$ ramp with single and two-phase faults.

h) Wide frequency range test 
This paper is a postprint of a paper submitted to and accepted for publication in IET Generation, Transmission and Distribution (GTD) and is subject to IET copyright. The copy of record is available at [http://digital-library.theiet.org/]" and the paper is available through IEEE Xplore at [http://ieeexplore.ieee.org/xpls/abs_all.jsp?arnumber=4907255]
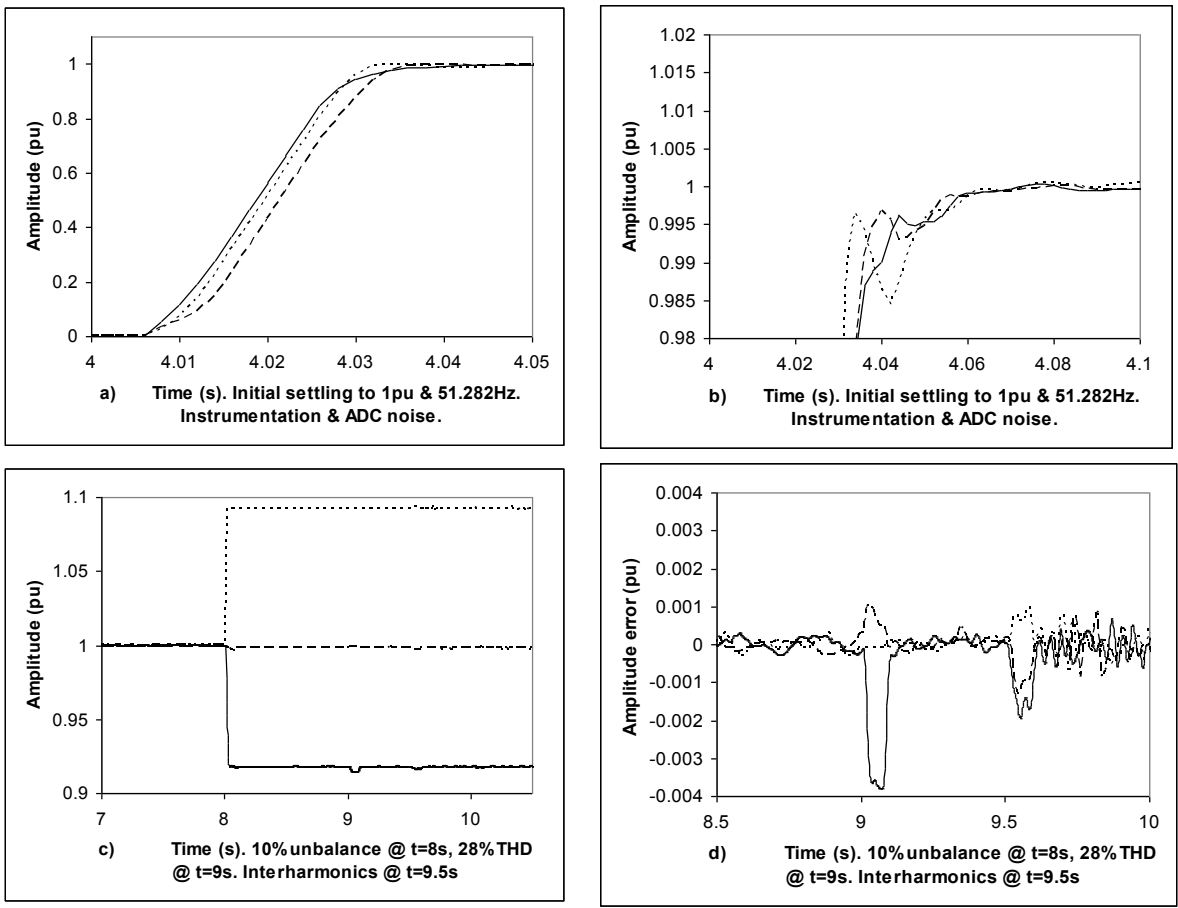

Fig. 10 Performance of FLL hybrid for amplitude measurement (three phases shown)

a) \& b) settling settling to $51.282 \mathrm{~Hz}$, with instrumentation and $A D C$ noise

c) $51.282 \mathrm{~Hz}$. Unbalance added at $\mathrm{t}=8 \mathrm{~s}, 28 \% \mathrm{THD}$ at $\mathrm{t}=9 \mathrm{~s}$, \& inter-harmonics at $\mathrm{t}=9.5 \mathrm{~s}$

d) as c) but amplitude errors (measured minus actual) shown, zoomed in to show detail. 
This paper is a postprint of a paper submitted to and accepted for publication in IET Generation, Transmission and Distribution (GTD) and is subject to IET copyright. The copy of record is available at [http://digital-library.theiet.org/]" and the paper is available through IEEE Xplore at [http://ieeexplore.ieee.org/xpls/abs_all.jsp?arnumber=4907255]

\section{$5 \quad$ References}

1 Johns, A. \& Salman, S. (1995). Digital protection for power systems. IEE Power Series 15, ISBN 086341303X

2 Petrovic, P., Marjanovic, S. \& Stevanovic, M. (2000). New algorithm for measuring $50 / 60 \mathrm{~Hz}$ AC values based on the usage of slow A/D converters. IEEE transactions on instrumentation and measurement, vol.49 issue 1. pp 166-171.

3 MATLAB Simulink SimPowerSystems blockset, "Discrete Fourier"

4 Aghazadeh, R. et al. (2005). New technique for frequency and amplitude estimation of power system signals. IEE Proceedings on generation, transmission and distribution, vol. 152, no. 3, pp 435-440.

$5 \quad$ Lopez, A. et al. (2008). Power system frequency measurement under nonstationary situations. IEEE Transactions on power delivery, vol. 22 no. 2, pp562-567.

6 Salcic, Z., \& Mikhael, R. (2000). A new method for instantaneous power system frequency measurement using reference points detection. Electric power systems research vol.55 issue 2 . pp 97-102.

7 Canteli, M. et. al. (2006). Three-phase adaptive frequency measurement based on Clarke's transformation. IEEE transactions on power delivery vol.21 issue 3. pp 1101-5.

8 Ignatova, V. et. al. (2005). Classification and characterisation of three-phase voltage dips by space vector methodology. International conference on future power systems. P 1600556.

9 Moore, P., Carranza, R. \& Johns, A. (1996). Model system tests on a new numeric method of power system frequency measurement. IEEE Transactions on power delivery, vol. 11 no. 2, pp 696-701.

10 Choi, J., Kim, Y. \& Kim, H. (2006). Digital PLL control for single-phase photovoltaic system. IEE proceedings on electric power applications, vol.153 issue 1. pp 40-6.

11 Lin, T. \& Domijan, A. (2005). On power quality indices and real time measurement. IEEE Transactions on power delivery, vol. 20 no. 4, pp 2552-2562.

12 Awad, H., Svensson, J. \& Bollen, M. (2005). Tuning software phase-locked loop for series-connected converters. IEEE transactions on power delivery, vol.20 issue 1. pp 300-8.

13 Han, B. \& Bae, B. (2006). Novel phase-locked loop using adaptive linear combiner. IEEE transactions on power delivery Han, vol.21 issue 1. pp 513-14.

14 Jovcic, D. (2003). Phase locked loop system for FACTS. IEEE transactions on power systems vol.18 issue 3. pp 1116-24.

15 MATLAB Simulink SimPowerSystems blockset, "Discrete Mean Value"

16 Roscoe, A. (2009). Measurement and control of microgrids at low frame rates to optimise security of supply. PhD thesis. University of Strathclyde.

17 MATLAB Simulink SimPowerSystems blockset, "Discrete Variable Transport Delay"

18 BSI, British Standards. (2000). Voltage characteristics of electricity supplies by public distribution systems. BS EN 50160:2000. ISBN 0580359255

19 TC1796 Tricore STKTC1796 starter kit: http://www.hitex.co.uk/shop/index1.html, accessed January 2009

20 Infineon Tricore family: http://www.infineon.com/cms/en/product, accessed January 2009

21 MVME5100: http://mototracker.atomicserver.co.uk/files/MVME5100\%20DS.pdf, accessed January 2009.

22 MVME5500: http://mototracker.atomicserver.co.uk/files/MVME5500\%20DS.pdf, accessed January 2009. 
This paper is a postprint of a paper submitted to and accepted for publication in IET Generation, Transmission and Distribution (GTD) and is subject to IET copyright. The copy of record is available at [http://digital-library.theiet.org/]" and the paper is available through IEEE Xplore at [http://ieeexplore.ieee.org/xpls/abs_all.jsp?arnumber=4907255]

23 BSI, British Standards. (2006). Electromagnetic compatibility (EMC) - Part 3-2: Limits for harmonic current emissions (equipment input current $\leq 16 \mathrm{~A}$ per phase). BS EN 61000-3-2:2006. ISBN 0580484572

24 BSI, British Standards. (2003). Electromagnetic compatibility (EMC) - Part 4-30: Testing and measurement techniques - Power quality measurement methods. BS EN 61000-4-30. ISBN 0580420817 